\title{
Molecular Characterization of Hsp47 in Grass Carp (Ctenopharyngodon idella) and Its Correlation with Type I Collagen in Response to Fish Aerobic Exercise
}

\author{
Xiao Liang ${ }^{1,2,+}$, Ying Wan ${ }^{1,+}$, Zhiyuan Shen ${ }^{1}$, Yanmei Liu ${ }^{1}$, Dapeng Li ${ }^{1} \mathbb{C}$, Li Li $^{1}{ }^{1}$, Rong Tang ${ }^{1}$ and Xi Zhang ${ }^{1, *}$ \\ 1 College of Fisheries, National Demonstration Center for Experimental Aquaculture Education, \\ Engineering Research Center of Green Development for Conventional Aquatic Biological Industry in the \\ Yangtze River Economic Belt, Ministry of Education, Hubei Provincial Engineering Laboratory for Pond \\ Aquaculture, Huazhong Agricultural University, Wuhan 430070, China; liangxiao1225@yeah.net (X.L.); \\ wany@webmail.hzau.edu.cn (Y.W.); Shenzhiyuan@webmail.hzau.edu.cn (Z.S.); \\ 311621Lym@webmail.hzau.edu.cn (Y.L.); ldp@mail.hzau.edu.cn (D.L.); foreverlili78@mail.hzau.edu.cn (L.L.); \\ tangrong@mail.hzau.edu.cn (R.T.) \\ 2 Institute of Hydrobiology, Zhejiang Academy of Agricultural Sciences, Hangzhou 310021, China \\ * Correspondence: zhangxi@mail.hzau.edu.cn; Tel.: +86-027-87282113 \\ + These authors contributed equally to this work.
}

\section{check for} updates

Citation: Liang, X.; Wan, Y.; Shen, Z.; Liu, Y.; Li, D.; Li, L.; Tang, R.; Zhang, X. Molecular Characterization of Hsp47 in Grass Carp (Ctenopharyngodon idella) and Its Correlation with Type I Collagen in Response to Fish Aerobic Exercise. Fishes 2021, 6, 17. https://doi.org/ $10.3390 /$ fishes 6020017

Academic Editor: Maria

Angeles Esteban

Received: 22 March 2021

Accepted: 19 April 2021

Published: 23 April 2021

Publisher's Note: MDPI stays neutral with regard to jurisdictional claims in published maps and institutional affiliations.

Copyright: (c) 2021 by the authors. Licensee MDPI, Basel, Switzerland. This article is an open access article distributed under the terms and conditions of the Creative Commons Attribution (CC BY) license (https:/ / creativecommons.org/licenses/by/ $4.0 /)$.

\begin{abstract}
Heat shock protein 47 (Hsp47) is a collagen-specific molecular chaperone that is indispensable for molecular maturation of collagen. In this study, $h s p 47$ and hsp47-like cDNAs were cloned and characterized in grass carp (Ctenopharyngodon idella). The cDNAs were 1212 and 1218 base pairs long, respectively, and included an open reading frame encoding 403 and 405 amino acids. The molecular phylogeny based on the deduced amino acid sequences indicated that the correct sequences of the hsp47 and hsp47-like cDNA were obtained and the deduced proteins clustered distinctly into teleost clades. Primary structure analysis and characterization of Hsp47 and Hsp47-like shared the basic structure and biofunctions of Hsp47 in vertebrates. The spatial pattern of gene expression revealed that $h s p 47$ and $h s p 47-l i k e$ were relatively ubiquitous in different tissues and highly expressed in heart and skin. The expression levels of $h s p 47$ and $h s p 47-l i k e$ and type I collagen mRNAs varied similarly in different tissues. Type I collagen content increased significantly with the increase of water velocity in the muscle of grass carp in response to aerobic exercise. Among the gene expression changes of $h s p 47, h s p 47-l i k e$, col1a1 and col1a2 in muscle that occurred in response to aerobic exercise, the change of type I collagen was most strongly correlated with $h s p 47$ expression. Additionally, colla1 showed the highest correlation with hsp47-like and colla2 showed the highest correlation with $h s p 47$. These findings suggest that grass carp Hsp47 and Hsp47-like are closely related to type I collagen synthesis. This study firstly suggests fish aerobic exercise can improve type I collagen content and Hsp47 gene expression in muscle of grass carp.
\end{abstract}

Keywords: grass carp; $h s p 47$; collagen; molecular cloning; gene expression

\section{Introduction}

Heat shock protein 47 (Hsp47) is a collagen-specific molecular chaperone that is essential for the correct folding of procollagen in the endoplasmic reticulum (ER) $[1,2]$. Hsp47 is encoded by the SerpinH1 gene and belongs to the serpin superfamily, but it does not inhibit serine proteases and it is specifically required for collagen folding [3,4]. Collagen is a major protein in animals, accounting for approximately $30 \%$ of total protein [5]. At present, 29 different types of collagen have been found and named (types I-XXIX) and they play different roles in tissues. Type I collagen is the most abundant collagen in vertebrate tissues and is a major component of the extracellular matrix [6,7]. Type I collagen consists of three $\alpha$ chains and it has a central triple-helical domain containing Gly-X-X triplets covering more than 300 residues. Hsp47 recognizes Gly-Xaa-Arg repeats on triple-helical 
procollagen and can prevent local unfolding or aggregate formation of procollagen $[8,9]$. Expression of Hsp47 is strongly correlated with expression of collagens in multiple types of cells and tissues [4]. However, little is known about the relationship between expression of Hsp47 and collagen in freshwater fish.

The grass carp (Ctenopharyngodon idella) is one of the most important cultured freshwater species in China $[10,11]$. Grass carp is rich in collagen, especially in the tissues of scales, skin and swim bladder [12,13]. Grass carp collagen can be commercially used as potential substitutes for mammalian collagens due to their comparatively high thermostability and fish collagen has two advantages than mammals, no risk of common human diseases and also avoiding religious objections [6,12-14]. The living environment, nutrition and physiological state of the grass carp may affect the properties of collagen to a certain extent $[15,16]$. The thermal stability of collagen from carp showed seasonal differences, with higher thermal stability in carp sacrificed in the summer [15]. Fish growth and physiological state are affected by external and internal factors and aerobic exercise in different water velocities has been reported to improve fish growth performance, innate immune response and disease resistance [10,11,17-19]. However, there is no relevant research on the effects of different water velocities on grass carp collagen and related genes.

Prior to this study, to our knowledge Hsp47 has not been investigated in this fish. We cloned and identified the cDNA sequence encoding Hsp47 and Hsp47-like in the grass carp, analyzed its functional characteristics and the phylogenetic relationships of its deduced amino acid sequence and observed the expression levels of $h s p 47$ and hsp47-like in different organs. The relationship between Hsp47 and type I collagen in grass carp was studied as well by evaluating their expression levels in different organs and the modulation of expression in muscle in response to fish aerobic exercise.

\section{Results}

\subsection{Molecular Cloning of Hsp47}

The nucleotide sequence of the grass carp $h s p 47$ was 1212 base pairs long and contained an open reading frame encoding 403 amino acids (GenBank accession MT657345) and that of hsp47-like was 1218 base pairs long and contained an open reading frame encoding 405 amino acids (GenBank accession MW263909).

\subsection{Structure Analysis and Characterization of Hsp47}

Figure 1 shows the deduced translation products of the $h s p 47$ and hsp47-like cDNA of grass carp. Hsp47 of grass carp is $91 \%$ identical to that of goldfish, $75 \%$ to zebrafish, $76 \%$ to rainbow trout, $72 \%$ to chicken and $67 \%$ to mouse and human. Hsp47-like is $76 \%$ identical to Hsp47 of grass carp and is 95\% identical to Hsp47-like of goldfish, 93\% to zebrafish and $83 \%$ to salmon. Multiple alignments revealed that the Hsp47 and Hsp47-like proteins of grass carp contained a typical hydrophobic signal sequence, an ER retention signal (RDEL) and a serine protease inhibitor signature sequence (FYADHPFIF), all of which are characteristic of Hsp47 in higher vertebrates [20,21] (Figure 1). In the deduced sequence, two potential Asn-X-Thr glycosylation sites (Asn-Ser-Thr (NST) and Asn-Val-Thr $(\mathrm{NVT})$ ) were found and they are similar to those in mammals $[3,20]$. Additionally, a third possible glycosylation site (Asn-Lys-Thr (NKT)) was found, which is similar to that found in zebrafish [20]. 


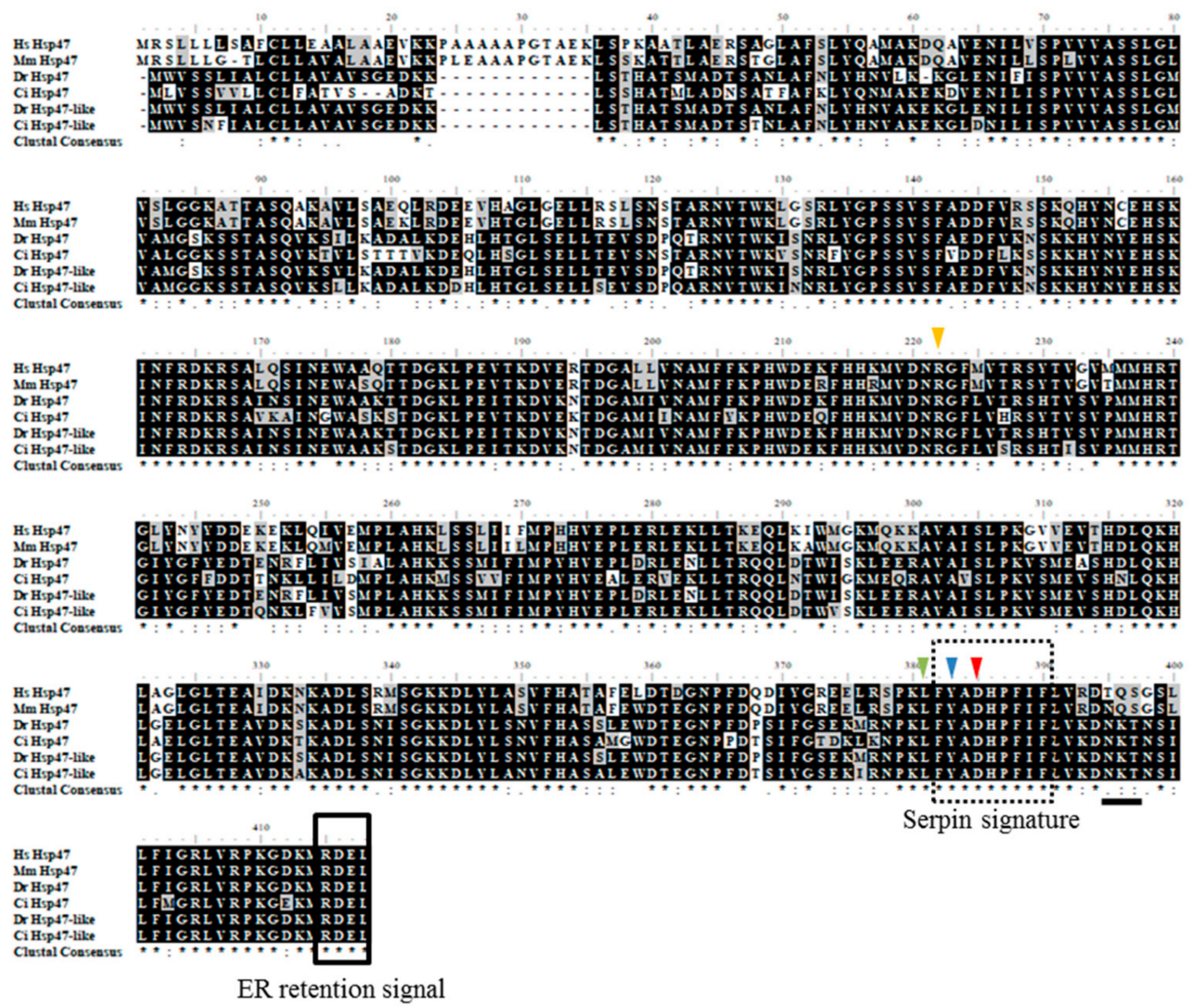

Figure 1. Deduced amino acid sequence of Hsp47 and Hsp47-like of grass carp and multiple sequence alignment with Hsp47s from other vertebrates. Identical residues between the sequences were indicated by the same color and asterisk $\left(^{*}\right)$ indicates the same amino acid. The sequence labeled serpin signature is boxed by a dotted line. The RDEL-ER retention signal sequence is indicated by the solid line box. The potential glycosylation site (Asn-X-Thr) is underlined. Residues representing $\operatorname{Arg}(\mathrm{R}), \mathrm{Leu}(\mathrm{L}), \operatorname{Tyr}(\mathrm{Y})$ and Asp (D) are indicated by different colored inverted triangles.

The three-dimensional (3D) models of Hsp47 and Hsp47-like were created using structural data from homology modeling (Figure 2). We selected the templates used for 3D structure prediction. The template for HSP47 and HSP47-like was "SERPIN PEPTIDASE INHIBITOR, CLADE H (HEAT SHOCK PROTEIN 47), MEMBER 1, (COLLAGEN BINDING PROTEIN 1)", whose accession number is 4au4.3. The structure of Hsp47 and Hsp47-like prediction is determined by X-ray crystallography with a resolution of $2.97 \AA$. The grass carp model was similar to the models for mammals and zebrafish [22], including the number and configuration of $\alpha$-helices and $\beta$-sheets, but one more $\beta$-sheet at the tail of Hsp47 and Hsp47-like was found in grass carp. 


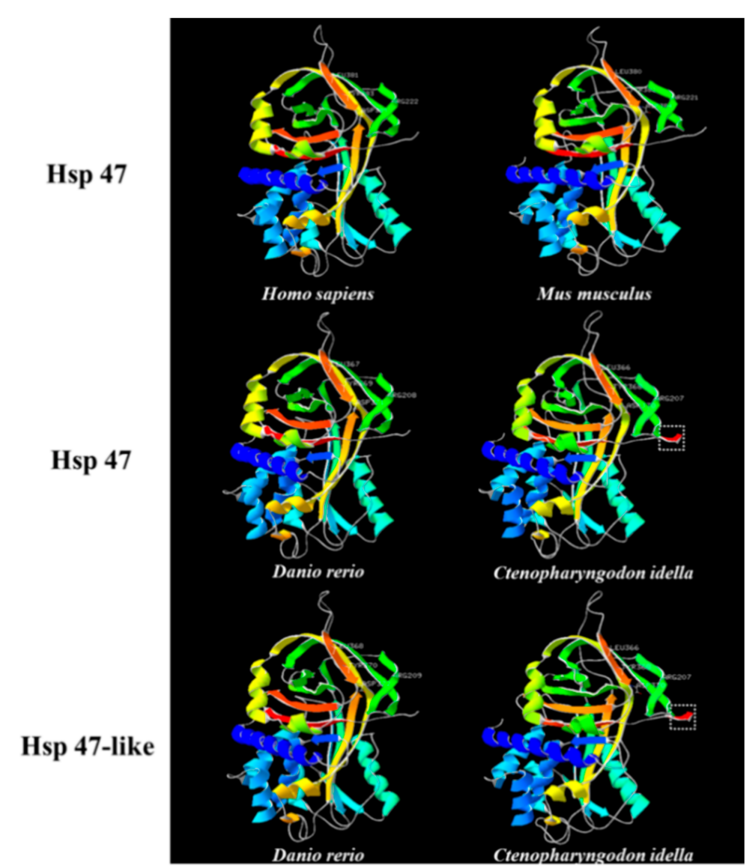

Figure 2. Structure analysis of Hsp47 and Hsp47-like of grass carp. A. Three-dimensional structures of the Hsp47 and HSp47-like deduced amino acid sequence predicted from humans (H. sapiens), zebrafish (D. rerio) and grass carp (C. idella). The $\alpha$-helices and $\beta$-sheets are represented by different colors. Residues representing Arg (R), Leu (L), Tyr (Y) and Asp (D) corresponding to Figure 1 are indicated in the structures. The extra $\beta$-sheet in grass carp is boxed by a dotted line.

\subsection{Phylogenetic Analysis}

To compare the relationship of the grass carp Hsp47 and Hsp47-like sequences to those of other vertebrates, a molecular phylogenetic tree was reconstructed based on deduced amino acids (Figure 3). Database accession numbers of the related sequences are shown in the figure. The tree indicated that the examined sequences for grass carp clustered as Hsp47 and Hsp47-like with high bootstrap support, which was consistent with the known phylogeny of vertebrate species. Grass carp Hsp47 and Hsp47-like were clustered into fish subgroups, with a close relationship with goldfish Hsp47.

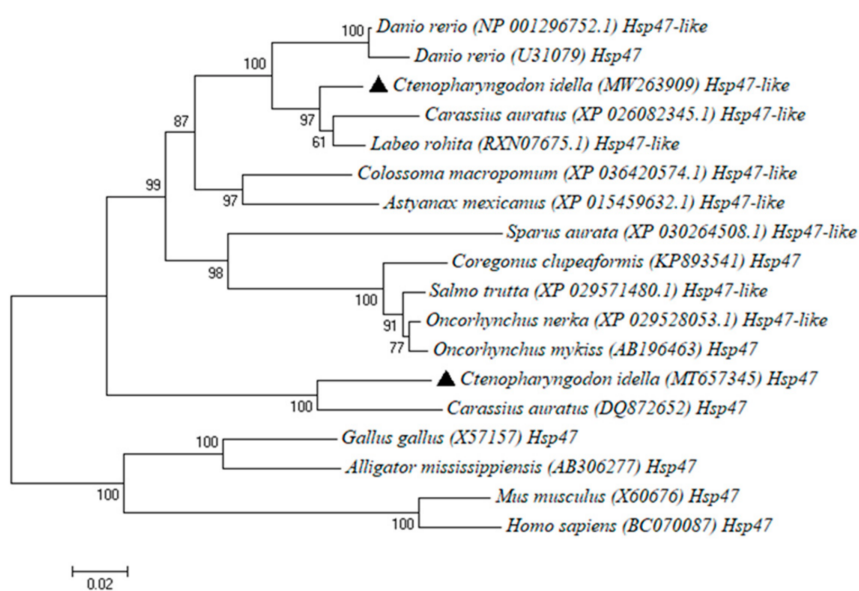

Figure 3. Phylogenetic analysis of Hsp47 and Hsp47-like of grass carp. Relationship between grass carp Hsp47 and Hsp47-like sequences and those of other vertebrates. The sequence for grass carp was predicted based on cDNA sequences obtained in the present study. The others were downloaded from the protein database and accession numbers are given in parentheses. Horizontal branch lengths are proportional to the amino acid substitution rate per site. Numbers represent frequencies (\%), with the tree topology presented after 1000 iterations. 


\subsection{Comparative Expression of Hsp47, Hsp47-Like and Type I Collagen Genes in Various Organs}

Figure 4 shows gene expression of $h s p 47, h s p 47-$ like and Type I collagen genes col1a1 and col1a 2 standardized by the expression of $\beta$-actin. All genes were widely expressed in the six examined organs, but they showed varied expression levels. The $h s p 47$ gene was highly expressed in the heart and skin, followed by the swim bladder, muscle and liver and expression was lowest in the intestine. The hsp47-like gene was highly expressed in the heart, followed by the swim bladder and skin and expression was low in other tissues. High expression of col1a1 and col1a2 was found in the skin, followed by the heart, muscle and liver and it was lowest in the intestine and swim bladder.

A

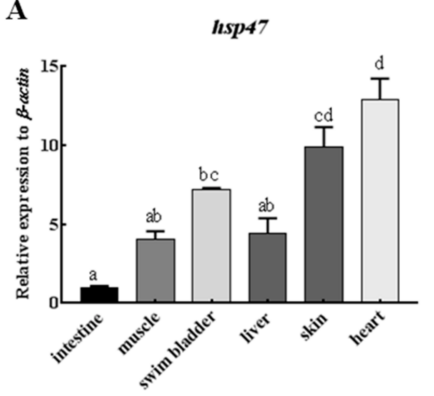

C

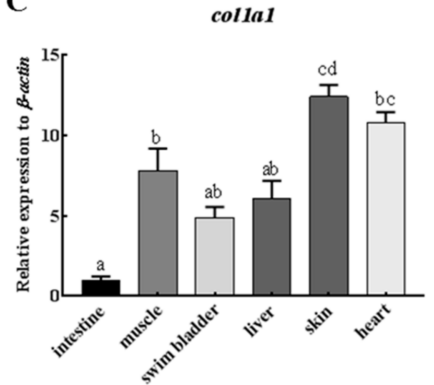

B

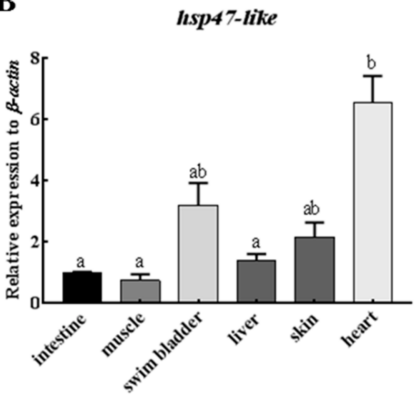

D

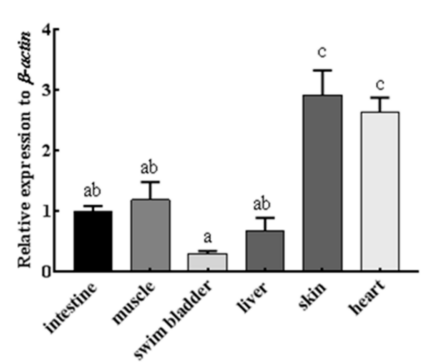

Figure 4. Relative expression of $h s p 47, h s p 47-l i k e$, col1a1 and col1a2 in various tissues of grass carp. Relative mRNA represents the mean \pm SE of samples. (A). relative expression of $h s p 47$; (B). relative expression of hsp47-like; (C). relative expression of col1a1; (D). relative expression of col1a2. Different lowercase letters above the bars indicate significant differences among tissues $(p<0.05)$.

\subsection{Effect of Aerobic Exercise on Expression of Muscle Hsp47, Hsp47-Like and Type I Collagen}

Transcriptional changes of $h s p 47, h s p 47-$ like and type I collagen genes colla1 and col1a2 in abdominal and tail muscle in response to aerobic exercise were detected (Figure 5A). In the muscle, colla1 transcript levels were significantly higher at water flow velocity of $0.5 \mathrm{bL} / \mathrm{s}$ compared with $0.0 \mathrm{bL} / \mathrm{s}$ and the transcript levels of colla 2 increased gradually with the increase of water flow velocity. Hsp47 mRNA expression was significantly higher at water flow velocity of 1.0 and $1.5 \mathrm{bL} / \mathrm{s}$ compared to that of $0 \mathrm{bL} / \mathrm{s}$, whereas Hsp47-like mRNA expression showed no significant changes among the groups. On the other hand, type I collagen content was significantly higher at water flow velocity of 1.0 and $1.5 \mathrm{bL} / \mathrm{s}$ compared to that of $0.0 \mathrm{bL} / \mathrm{s}$ (Figure 5B). In addition, correlations between hsp47, hsp 47-like expression levels and those of type I collagen genes were analyzed (Figure 5C): col1a1 showed the highest correlation with hsp47-like and col1a2 showed the highest correlation with $h s p 47$. Overall, type I collagen content was most strongly correlated with $h s p 47$ expression (Figure 5C). 


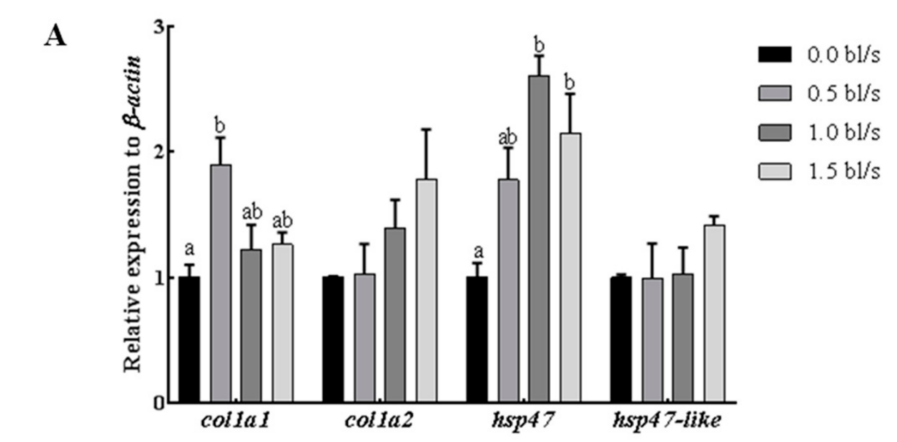

B

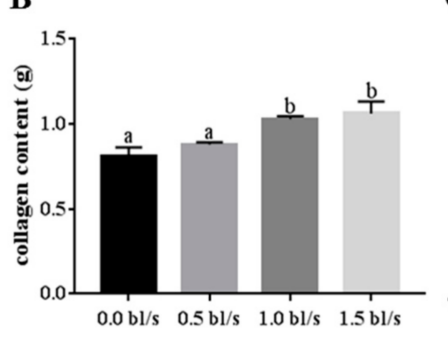

C

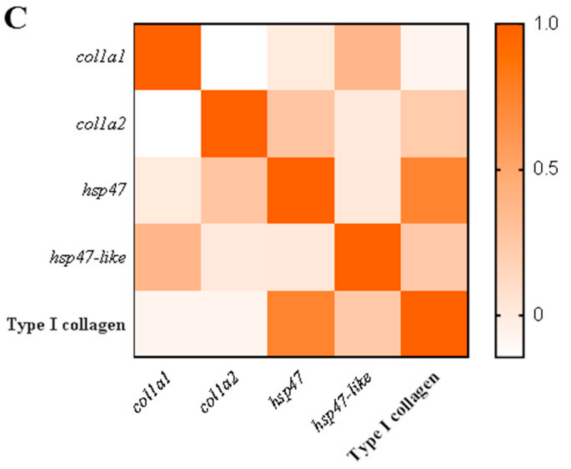

Figure 5. Effects of aerobic exercise on relative expression of genes, type I collagen content and the correlation between genes as well the collagen in muscle of grass carp cultured in four different water velocities $(0.0,0.5,1.0$ and $1.5 \mathrm{bL} / \mathrm{s})$. (A). relative expression of genes $h s p 47$, hsp 47-like, col1a1 and col1a2 in muscle; (B). type I collagen content; (C). correlation among hsp47, hsp47-like, col1a1 and col1a2 expression and type I collagen content in muscle. Different lowercase letters above the bars indicate significant differences among tissues $(p<0.05)$.

\section{Discussion}

In this study, we identified the $h s p 47$ and hsp47-like genes from the economically important grass carp for the first time. The Hsp47 and Hsp47-like amino acid sequences in grass carp share a high degree of identity with those of other vertebrates. The open reading frame length is consistent with that of the $h s p 47$ gene in goldfish [21], but is a little bit shorter than those of zebrafish and rainbow trout [20,23]. The typical hydrophobic signal sequence, an ER retention signal (RDEL) and a serine protease inhibitor signature sequence (FYADHPFIF) were well conserved in the amino acid sequence deduced in the present study. The Hsp47 and Hsp47-like deduced sequences also contained three potential Asn-XThr glycosylation sites, two of which are similar to those found in chickens and mammals and the third is similar to that found in zebrafish, which is absent in the mammals $[3,20]$. These observations indicated that Hsp47 and Hsp47-like in grass carp likely share the same basic biofunctions of Hsp47 present in other vertebrates [20,21].

Hsp47 transiently binds to procollagen in the ER, dissociates in the cis-Golgi or ERGolgi intermediate compartment in a pH-dependent manner and is then transported back to the ER via its RDEL retention sequence [4]. Based on the synthetic collagen model presented in previous research [22], Hsp47 and Hsp47-like residues in grass carp are predicted to interact with Asp370 and Arg207 at the Yaa ${ }^{0}$ position and the $\mathrm{Yaa}^{-3}$ position of the Yaa ${ }^{-3}$-Gly-Xaa-Arg sequence of collagen. Additionally, Leu366 and Tyr368 are predicted to be involved in several hydrophobic interactions. These residues suggest high conservation from fish to mammals. The 3D structure of grass carp Hsp47 and Hsp47-like are very similar to those of humans and zebrafish [22]. Interestingly, grass carp has one more $\beta$-sheet at the tail, which may indicate that grass carp Hsp47 and Hsp47-like have additional properties, which should be further studied in the future [20,21].

The phylogenetic tree based on the Hsp47 and Hsp47-like deduced amino acid sequences obtained in this study indicated a clear affiliation of Hsp47 and Hsp47-like in 
vertebrates. A similar phylogenetic tree structure of zebrafish was found in a previous study [20]. Both grass carp Hsp47 and Hsp47-like were clustered into fish subgroups and shared a close relationship with goldfish, which was consistent with the close evolutionary relationship of grass carp and goldfish.

Tissue expression of grass carp hsp 47, hsp 47-like and type I collagen genes detected by qRT-PCR revealed varied expression levels in different organs in this study. Gene expression levels of $h s p 47$ and $h s p 47$-like showed similar changing trends with those of col1a1 and col1a2 in different organs. The highest expression of $h s p 47$ and type I collagen genes occurred in the heart and skin, while the highest expression of hsp47-like is higher in skin and swim bladder. Grass carp skin was reported to contain an abundance of type I collagen [7,14], which is a fibril-forming collagen usually consisting of two identical a1-chains and an a2-chain [2,6,24]. Hsp47 is essential in the process of collagen synthesis and it is strongly correlated with expression of type I collagen in multiple tissues [2,4]. In this study, similar conclusions and trends were found, as gene expression levels of $h s p 47$ and hsp47-like were correlated to a certain extent with the expression of colla1 and col1a2 in multiple tissues. However, protein expression of Hsp47 and type I collagen in different organs was not analyzed in this study, collagen and Hsp47 abundance and functional relevance are limited to some extent since mRNA levels do not always correlate well with protein levels.

Recently, it has also been found that aerobic exercise, as a mechanism to improve fish culture yield, has a good effect on muscle quality [25]. Moderate sustained aerobic exercise can improve muscle growth and cellularity of gilthead sea bream (Sparus aurata L.) [26]. Exercise training can increase protein precipitation and polyunsaturated fatty acid content in white muscle of Barbodes polylepis, while decrease fat content and change fatty acid distribution in muscle of salmon (O. tshawytscha) $[27,28]$. Research showed that skeletal muscle causes complex temporal and spatial adaptive molecular responses in response to exercise in zebrafish [29]. Exercise-induced contractile activity promotes a coordinated adaptive response that leads to myogenesis, satellite cell activation and increases muscle mass by hypertrophy and vascularization by angiogenesis in adult zebrafish [30]. Collagen acts as a key component part of muscle in fish, but few studies are focused on the effects of different water velocities on collagen and its regulatory mechanism.

Therefore, we studied the relationship between Hsp47 and type I collagen in grass carp by evaluating their expression levels in the muscle of grass carp during aerobic exercise. Aerobic exercise has been reported to have an important effect on fish muscle growth and type I collagen plays an important role in muscle, as it is the major constituent of fish intramuscular connective tissue $[17,18,31,32]$. In this study, type I collagen content of grass carp muscle increased significantly with increased water flow velocity, which suggested that aerobic exercise helps to improve the collagen content of muscle. Additionally, type I collagen content was much more strongly correlated with $h s p 47$ expression than with colla1 and col1a2 expression in the muscle during aerobic exercise, which suggested that Hsp47 is crucially important in collagen synthesis in grass carp. In the process of type I collagen synthesis, after the formation of two $\alpha 1$-chains and one $\alpha 2$-chain, Hsp47 is specifically required for collagen folding and acceleration of triple-helix formation of collagen $[2,33]$. In the study of $h s p 47-k n o c k e d$ out fibroblasts, procollagens were ultimately degraded by autophagy and the secretion of type I collagen was slow $[4,34]$. On the other hand, the small molecule HSP47 inhibitor had ability to prevent collagen overexpression and inhibit the viability and migration of fibroblasts in vitro [35]. However, most of these Hsp47 studies focused on mammals and this study firstly confirmed the crucial correlation between Hsp47 and collagen in grass carp. 


\section{Materials and Methods}

\subsection{Experimental Fish and Sampling}

Grass carp ( $93 \pm 7 \mathrm{~g}$ ) were procured from the Huanggang Bairong Fish Farm and transported to the aquaculture center of Huazhong Agricultural University, China. The fish were allowed to adapt to the experimental environment for 1 month. Five fish were randomly selected for $h s p 47$ cloning and to evaluate the tissue distribution of $h s p 47$. Each fish was deeply anesthetized in 2-phenoxyethanol solution. The skin, swim bladder, muscle, liver, intestine and heart tissue were collected and placed in liquid nitrogen. These tissue samples were stored at $-80{ }^{\circ} \mathrm{C}$ until RNA extraction. For the fish aerobic exercise experiment, grass carp were cultured in four different water velocities 0.0, 0.5, 1.0 and 1.5 body length $/ \mathrm{s}(\mathrm{bL} / \mathrm{s})$ for 70 days. Each group had 3 parallel tanks and 14 fish in every tank. The circular polyethylene tanks ( $280 \mathrm{~L}$ ) was used in this study and the culture experimental system with circular water flow was shown in Figure 6. The fish were fed to satiation with identical feeds (Haid Group Co., Ltd., Wuhan, China). After the culture experiment, 5 fish from each group were randomly selected and their muscle were collected and processed as previously described.
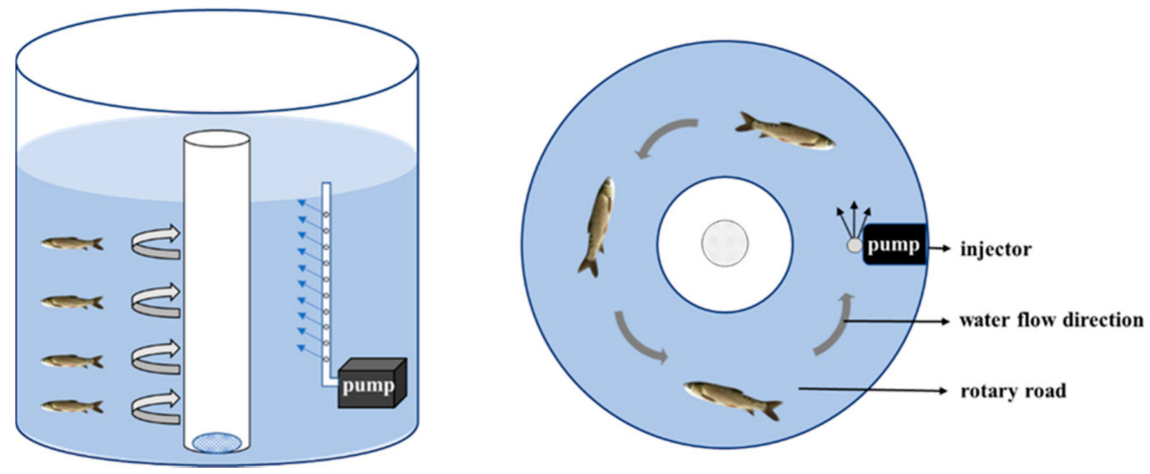

Figure 6. The scheme of culture experimental system with circular water flow.

\subsection{Cloning of Hsp47 in Grass Carp}

Total RNA was isolated from tissues using TRIpure Reagent (Aidlab, Beijing, China) according to the manufacturer's instructions. Qualities and Quantities of extracted total RNA were assured by gel electrophoresis and ultraviolet spectrophotometry (Nanodrop 2000, Thermo Scientific, USA). Total RNA was reverse-transcribed into cDNA using the PrimeScript RT Reagent Kit with gDNA Eraser (TaKaRa, Shuzo, Japan). The $3^{\prime}$ and $5^{\prime}$ ends of cDNAs were synthesized using the SMART RACE Kit (Clontech, CA, USA) according to the manufacturer's protocol. Several oligonucleotide primers were used for PCR, which were designed from nucleotide sequences in the conserved regions of $h s p 47$ from several vertebrate taxa obtained from the National Center for Biotechnology Information (NCBI) and sequences newly obtained in the present study. Another Hsp47-like was found by the blast of Hsp47 from the genome database of grass carp (http://bioinfo.ihb.ac.cn/ $\mathrm{gcgd} / \mathrm{php} /$ index.php (accessed on 8 October 2020) and checked using the primer. The PCR primers for cloning experiment are shown in Table 1. PCR products were subcloned using the pMD-19T vector (Takara, Dalian, China), transformed into Escherichia coli DH5 $\alpha$ competent cells and the positive clones were sequenced. 
Table 1. PCR primers used for cloning and gene expression studies.

\begin{tabular}{|c|c|}
\hline Primers & Primer Sequence $\left(5^{\prime}-3^{\prime}\right)$ \\
\hline hsp47 01-F1 & TGAGCTGGGTCTGACTGAAG \\
\hline hsp47 01-R1 & CAAAAGGATGGTCGGCGTAG \\
\hline hsp47 02-F1 & GCCCAGTTCTGTTAGCTTCG \\
\hline hsp 47 02-R1 & ACCACGATTGTCCACCATCT \\
\hline hsp47 03-F1 & AGATGGTGGACAATCGTGGT \\
\hline hsp47 03-R1 & CAAAAGGATGGTCGGCGTAG \\
\hline hsp $475^{\prime}$ race-outer & CCATCTTGTGATGGAACTGCTCATCC \\
\hline hsp $475^{\prime}$ race-inner & GCGCTTGTCACGGAAGTTTATTTTGG \\
\hline hsp $473^{\prime}$ race-outer & CAGGGAAGAAAGACCTCTACCTGTCCAATG \\
\hline hsp $473^{\prime}$ race-inner & GAAATCCACCCGATACGAGCATCTTTGG \\
\hline hsp47-like F1 & TCTCTACCACAATGTCGCCA \\
\hline hsp47-like R1 & CGCTCACCTCGCTCAGAA \\
\hline$h s p 47-\mathrm{qF}$ & TGAGCTGGGTCTGACCGAAG \\
\hline$h s p 47-\mathrm{qR}$ & TGAAGGGATGGTCAGCGTAG \\
\hline hsp47-like-qF & TCTCTACCACAATGTCGCCA \\
\hline hsp47-like-qR & CGCTCACCTCGCTCAGAA \\
\hline col1 $\alpha 1-\mathrm{qF}$ & GCATGGGGCAAGACAGTCA \\
\hline $\operatorname{col} 1 \alpha 1-\mathrm{qR}$ & ACGCACACAAACAATCTCAAGT \\
\hline $\operatorname{col} 1 \alpha 2-\mathrm{qF}$ & ACATTGGTGGCGCAGATCA \\
\hline $\operatorname{col} 1 \alpha 2-\mathrm{qR}$ & TCTCCGATAGAGCCCAGCTT \\
\hline$\beta$-actin- $\mathrm{qF}$ & GCCGTGACCTGACTGACT \\
\hline$\beta$-actin-qR & CAAGACTCCATACCCAAGAA \\
\hline
\end{tabular}

\subsection{Characterization and Phylogenetic Analysis of Hsp47 in Grass Carp}

Amino acid sequences of Hsp47 and Hsp47-like were translated from the cloned nucleotide sequences using Alignx analysis and the protein identity was searched using BLAST. A phylogenetic tree of the deduced amino acid sequences of Hsp47 and Hsp47-like was constructed using the neighbor-joining method (bootstrap method: 1000 replications) in MEGA 6 and amino acid sequences of the Hsp47s and Hsp47-likes from several vertebrate species were obtained from NCBI. Functional domains of grass carp Hsp47 and Hsp47-like were predicted by multiple alignments with other vertebrate species using data from previous studies [3,20]. The 3D structures of Hsp47 and Hsp47-like were predicted by SWISS-MODEL and a 3D model was constructed using tools for comparative protein structure modeling [36].

\subsection{Gene Expression and Collagen Content Analysis}

Gene expression levels of $h s p 47, h s p 47-l i k e$ and type I collagen genes colla1 and col1a2 in tissues collected before were measured using quantitative real-time (qRT)-PCR and $\beta$-actin was used as the reference gene to normalize the expression level. The reverse transcribed cDNA was used as the template and the qRT-PCR assay was performed using Hieff ${ }^{\circledR}$ qPCR SYBR Green Master Mix (YEASEN, Shanghai, China) on a Roche Light Cycler 480 machine (Roche, Sussex, UK). The specific amplification for each primer pair was confirmed by dissociation curve analysis at the end of the PCR reaction. The experiment was conducted in triplicate. Primers for qRT-PCR are listed in Table 1. Collagen content of muscle was measured using the Fish Collagen Type I (ColI) Elisa Kit (Nanjing Jiancheng Biochemical Corporation, Nanjing, China).

\subsection{Statistical Analysis}

Statistical analyses were performed using SPSS 25.0 (IBM, Armonk, NY, USA) and the results are shown as mean \pm standard error of the mean (SE). One-way analysis of variance followed by Duncan's multiple comparison tests was used to compare the expression of each gene among different tissues and the gene expression between grass carp cultured in different water velocities. Normality and variance uniformity were verified. Significant 
differences were confirmed at $p<0.05$. The correlation between genes and collagen content was analyzed using the Pearson correlation coefficient.

\section{Conclusions}

Molecular characterization of Hsp47 and its correlation with type I collagen in response to aerobic exercise in grass carp was studied. Primary structure analysis and characterization of Hsp47 and Hsp47-like suggested that they share the conserved structural features and basic biofunctions of Hsp47 proteins. In the phylogenetic tree analysis, the grass carp Hsp47 proteins clustered in the teleost clade and were closely aligned with those of goldfish. Grass carp hsp47 expression showed tissue variations, with the highest expression in skin and heart. Transcriptional changes of Hsp47 in different organs showed similar changing trends with those of type I collagen. In the muscle of grass carp during aerobic exercise, type I collagen content was much more strongly correlated with the expression of $h s p 47$ than with the expression of col1a1 and col1a2, which suggested that Hsp47 is crucially important in collagen synthesis in grass carp. To our knowledge, this is the first study to report the cloning of $h s p 47$ and the correlation between Hsp47 and collagen in grass carp and the results provide basic and general information about Hsp47 in fish. This study firstly suggests fish aerobic exercise can improve type I collagen content and Hsp47 gene expression in muscle of grass carp.

Author Contributions: Conceptualization, X.Z.; Data curation, X.L. and Y.W.; Funding acquisition, X.Z. and D.L.; Investigation, Y.W., Z.S. and Y.L.; Methodology, X.L., Y.W. and X.Z.; Supervision, X.Z.; Writing—original draft, X.L. and X.Z.; Writing—review \& editing, X.L., D.L., L.L., R.T. and X.Z. All authors have read and agreed to the published version of the manuscript.

Funding: This work was financially supported by the National Natural Science Foundation of China (31902390), the National Key Research and Development Program of China (2019YFD0900303) and the Earmarked Fund for China Agriculture Research System (CARS-45).

Institutional Review Board Statement: In the present study, all procedures were performed in accordance with the "Guidelines for Experimental Animals" of the Ministry of Science and Technology (Beijing, China) and they were approved by the Institutional Animal Care and Use Committees of Huazhong Agricultural University (HZAUFI-2020-001).

Conflicts of Interest: The authors declare no conflict of interest. The work has not been published previously.

\section{References}

1. Nakai, A.; Satoh, M.; Hirayoshi, K.; Nagata, K. Involvement of the stress protein HSP47 in procollagen processing in the endoplasmic reticulum. J. Cell Biol. 1992, 117, 903-914. [CrossRef] [PubMed]

2. Ito, S.; Nagata, K. Roles of the endoplasmic reticulum-resident, collagen-specific molecular chaperone Hsp47 in vertebrate cells and human disease. J. Biol. Chem. 2019, 294, 2133-2141. [CrossRef] [PubMed]

3. Hirayoshi, K.; Kudo, H.; Takechi, H.; Nakai, A.; Iwamatsu, A.; Yamada, K.M.; Nagata, K. HSP47: A tissue-specific, transformationsensitive, collagen-binding heat shock protein of chicken embryo fibroblasts. Mol. Cell Biol. 1991, 11, 4036-4044. [CrossRef] [PubMed]

4. Ito, S.; Nagata, K. Biology of Hsp47 (serpin H1), a collagen specific molecular chaperone. Semin. Cell Dev. Biol. 2017, 62, 142-151. [CrossRef]

5. Muyonga, J.H.; Cole, C.G.B.; Duodu, K.G. Characterization of acid soluble collagen from skins of young and adult Nile perch (Lates niloticus). Food Chem. 2004, 85, 81-89. [CrossRef]

6. Zhang, X.; Adachi, S.; Ura, K.; Takagi, Y. Properties of collagen extracted from Amur sturgeon Acipenser schrenckii and assessment of collagen fibrils in vitro. Int. J. Biol. Macromol. 2019, 137, 809-820. [CrossRef]

7. Zhu, S.; Yuan, Q.; Yang, M.; You, J.; Yin, T.; Gu, Z.; Hu, Y.; Xiong, S. A quantitative comparable study on multi-hierarchy conformation of acid and pepsin-solubilized collagens from the skin of grass carp (Ctenopharyngodon idella). Mater. Sci. Eng. C 2019, 96, 446-457. [CrossRef]

8. Jain, N.; Brickenden, A.; Lorimer, I.; Ball, E.H.; Sanwal, B.D. Interaction of procollagen I and other collagens with colligin. Biochem. J. 1994, 304, 61-68. [CrossRef]

9. Koide, T.; Takahara, Y.; Asada, S.; Nagata, K. Xaa-Arg-Gly triplets in the collagen triple helix are dominant binding sites for the molecular chaperone HSP47. J. Biol. Chem. 2002, 277, 6178-6182. [CrossRef] 
10. Zhao, H.; Xia, J.; Zhang, X.; He, X.; Li, L.; Tang, R.; Chi, W.; Li, D. Diet affects muscle quality and growth traits of grass carp (Ctenopharyngodon idellus): A comparison between grass and artificial feed. Front. Physiol. 2018, 9, 283. [CrossRef]

11. Zhang, X.; Wang, J.; Tang, R.; He, X.; Li, L.; Takagi, Y.; Li, D. Improvement of muscle quality of grass carp (Ctenopharyngodon idellus) with a bio-floating bed in culture ponds. Front. Physiol. 2019, 10, 683. [CrossRef]

12. Liu, D.; Zhang, X.; Li, T.; Yang, H.; Zhang, H.; Regenstein, J.; Zhou, P. Extraction and characterization of acid- and pepsin-soluble collagens from the scales, skins and swim-bladders of grass carp (Ctenopharyngodon idella). Food Biosci. 2015, 9, 68-74. [CrossRef]

13. Hu, J.H.; Li, T.C.; Liu, X.Y.; Liu, D.S. Seasonal variation of acid-soluble collagens extracted from the swim bladders and skins of bighead carp (Hypophthalmichthys nobilis) and grass carp (Ctenopharyngodon idella). Food Biosci. 2016, 15, 27-33. [CrossRef]

14. Zhang, X.; Zhang, H.; Toriumi, S.; Ura, K.; Takagi, Y. Feasibility of collagens obtained from bester sturgeon Huso huso $\times$ Acipenser ruthenus for industrial use. Aquaculture 2020, 529, 735641. [CrossRef]

15. Duan, R.; Zhang, J.J.; Du, X.Q.; Yao, X.C.; Konno, K. Properties of collagen from skin, scale and bone of carp (Cyprinus carpio). Food Chem. 2009, 112, 702-706. [CrossRef]

16. Ma, Y.B.; Zhang, J.X.; Zhou, X.Q.; Jiang, W.D.; Wu, P.; Liu, Y.; Kuang, S.Y.; Tang, L.; Feng, L. Effect of tea polyphenols on flavour, healthcare components, physicochemical properties, and mechanisms of collagen synthesis in growing grass carp (Ctenopharyngodon idella) muscle. Aquaculture 2021, 534, 736237. [CrossRef]

17. Liu, G.Y.; Wu, Y.J.; Qin, X.H.; Shi, X.T.; Wang, X.L. The effect of aerobic exercise training on growth performance, innate immune response and disease resistance in juvenile Schizothorax prenanti. Aquaculture 2018, 486, 18-25. [CrossRef]

18. Kirk, M.A.; Caudill, C.C.; Tonina, D.; Syms, J.C. Effects of water velocity, turbulence and obstacle length on the swimming capabilities of adult Pacific lamprey. Fish. Manag. Ecol. 2016, 23, 356-366. [CrossRef]

19. Gutierrez, P.T.; Almeida, F.L.; Carani, F.R.; Vechetti, I.J.; Padovanic, C.R.; Salomão, R.A.S. Rearing temperature induces changes in muscle growth and gene expression in juvenile pacu (Piaractus mesopotamicus). Comp. Biochem. Phys. B 2014, 169, 31-37. [CrossRef]

20. Pearson, D.S.; Kulyk, W.M.; Kelly, G.M.; Krone, P.H. Cloning and characterization of a cDNA encoding the collagen-binding stress protein hsp47 in zebrafish. DNA Cell Biol. 1996, 15, 263-272. [CrossRef]

21. Wang, J.; Wei, Y.; Li, X.; Cao, H.; Xu, M.; Dai, J. The identification of heat shock protein genes in goldfish (Carassius auratus) and their expression in a complex environment in Gaobeidian Lake, Widmer Beijing, China. Comp. Biochem. Phys. C 2007, 145, 350-362. [CrossRef] [PubMed]

22. Widmer, C.; Gebauer, J.M.; Brunstein, E.; Rosenbaum, S.; Zaucke, F.; Drögemüller, C.; Leeb, T.; Baumann, U. Molecular basis for the action of the collagen-specific chaperone Hsp47/SERPINH1 and its structure-specific client recognition. Proc. Natl. Acad. Sci. USA 2012, 109, 13243-13247. [CrossRef] [PubMed]

23. Ojima, N.; Yamashita, M.; Watabe, S. Comparative expression analysis of two paralogous Hsp70s in rainbow trout cells exposed to heat stress. Biochim. Biophys. Acta 2005, 168, 99-106. [CrossRef] [PubMed]

24. Zhang, X.; Azuma, N.; Hagihara, S.; Adachi, S.; Ura, K.; Takagi, Y. Characterization of type I and II procollagen $\alpha 1$ chain in Amur sturgeon (Acipenser schrenckii) and comparison of their gene expression. Gene 2016, 579, 8-16. [CrossRef]

25. Vélez, E.J.; Sheida, A.; Dorothy, V.; Cristina, S.; Eamail, L.; Albert, S.M.; Isabel, N.; Joaquim, G.; Encarnacion, C.; Silvia, M.L. Proteolytic systems' expression during myogenesis and transcriptional regulation by amino acids in gilthead sea bream cultured muscle cells. PLoS ONE 2017, 12, e0187339. [CrossRef]

26. Ibarz, A.; Felip, O.; Fernández-Borràs, J.; Martín-Pérez, M.; Blasco, J.; Torrella, J.R. Sustained swimming improves muscle growth and cellularity in gilthead sea bream. J. Comp. Physiol. B 2011, 181, 209-217. [CrossRef]

27. Kiessling, A.; Pickova, J.; Eales, J.G.; Dosanjh, B.; Higgs, D. Age, ration level, and exercise affect the fatty acid profile of chinook salmon (Oncorhynchus tshawytscha) muscle differently. Aquaculture 2004, 243, 345-356. [CrossRef]

28. Zhu, Z.M.; Song, B.L.; Lin, X.T.; Xu, Z.N. Effect of sustained training on glycolysis and fatty acids oxidation in swimming muscles and liver in juvenile tinfoil barb Barbonymus schwanenfeldii (bleeker, 1854). Fish. Physiol. Biochem. 2016, 42, 1-11. [CrossRef]

29. Lemoine, C.; Craig, P.M.; Dhekney, K.; Kim, J.J.; Mcclelland, G.B. Temporal and spatial patterns of gene expression in skeletal muscles in response to swim training in adult zebrafish (Danio rerio). J. Comp. Physiol. B 2010, 180, 151-160. [CrossRef]

30. Palstra, A.P.; Rovira, M.; Rizo-Roca, D.; Torrella, J.; Spaink, H.P.; Planas, J.V. Swimming-induced exercise promotes hypertrophy and vascularization of fast skeletal muscle fibres and activation of myogenic and angiogenic transcriptional programs in adult zebrafish. BMC Genom. 2014, 15, 1136. [CrossRef]

31. Cheng, J.H.; Sun, D.W.; Han, Z.; Zeng, X.A. Texture and structure measurements and analyses for evaluation of fish and fillet freshness quality: A review. Comp. Rev. Food Sci. 2014, 13, 52-61. [CrossRef]

32. Zhang, X.; Shen, Z.; Qi, T.; Xi, R.; Liang, X.; Li, L.; Tang, R.; Li, D. Slight increases in salinity improve muscle quality of grass carp (Ctenopharyngodon idellus). Fishes 2021, 6, 7. [CrossRef]

33. Murakami, S.; Toda, Y.; Seki, T.; Munetomo, E.; Kondo, Y.; Sakurai, T.; Furukawa, Y.; Matsuyama, M.; Nagate, T.; Hosokawa, N.; et al. Heat shock protein (HSP) 47 and collagen are upregulated during neointimal formation in the balloon-injured rat carotid artery. Atherosclerosis 2001, 157, 361-368. [CrossRef]

34. Kawasaki, K.; Ushioda, R.; Ito, S.; Ikeda, K.; Masago, Y.; Nagata, K. Deletion of the collagen-specific molecular chaperone hsp47 causes endoplasmic reticulum stress-mediated apoptosis of hepatic stellate cells. J. Biol. Chem. 2015, 290, 3639-3646. [CrossRef] 
35. Miyamura, T.; Sakamoto, N.; Kakugawa, T.; Taniguchi, H.; Akiyama, Y.; Okuno, D.; Moriyama, S.; Hara, A.; Kido, T.; Ishimoto, H.; et al. Small molecule inhibitor of HSP47 prevents pro-fibrotic mechanisms of fibroblasts in vitro. Biochem. Bioph. Res. Co. 2020, 530, 561-565. [CrossRef]

36. Arnold, K.; Bordoli, L.; Kopp, J.; Schwede, T. The SWISS-MODEL workspace: A web-based environment for protein structure homology modelling. Bioinformatics 2006, 22, 195-201. [CrossRef] 